\title{
aMAP Score as a Predictor for Long-Term Outcomes in Patients with HBV-Related Acute-on-Chronic Liver Failure
}

\section{Yunqing Sun (iD* \\ Zhuohong Li (iD)* \\ Guichan Liao \\ Muye Xia \\ Xuwen Xu (iD \\ Shaohang Cai $(\mathbb{D}$ \\ Jie Peng}

Department of Infectious Diseases, Nanfang Hospital, Southern Medical University, Guangzhou, Guangdong

Province, People's Republic of China

*These authors contributed equally to this work
Correspondence: Jie Peng; Shaohang Cai Email pjiel38@I63.com;

shaohangcai@foxmail.com
Background and Aim: The long-term outcomes of patients with hepatitis B virus-related acute-on-chronic liver failure (HBV-ACLF) remain not well known. This study aimed to investigate whether aMAP score can predict re-hospitalization, hepatocellular carcinoma (HCC) occurrence and long-term mortality in patients with HBV-ACLF.

Methods: A total of 82 patients diagnosed with HBV-ACLF and survived over 6 months were enrolled. The median follow-up period was 105 (75.9, 134.1) months. The Cox proportional hazards or logistic regression analysis was used to determine independent risk factors. Cumulative incidence of HCC and survival rate were evaluated using Kaplan-Meier analysis. Results: Multivariate analysis identified that the aMAP risk score was an independent predictor of re-hospitalization (odds ratio $[\mathrm{OR}]=1.112,95 \%$ confidence interval [CI]: 1.021-1.211, $\mathrm{p}=$ 0.015 ), hepatocellular carcinoma occurrence (hazards ratio $[\mathrm{HR}]=2.277,95 \% \mathrm{CI}: 1.014-5.114$, $\mathrm{p}=0.046)$ and mortality $(\mathrm{HR}=1.366,95 \% \mathrm{CI}: 1.040-1.794, \mathrm{p}=0.025)$. High-risk aMAP scores were associated with higher risk of HCC occurrence and mortality.

Conclusion: A higher aMAP score was an independent risk predictor of re-hospitalization, HCC occurrence and mortality, respectively, in HBV-ACLF patients who survived over 6 months, which can be applicable for early risk stratification and clinical decision.

Keywords: acute-on-chronic liver failure, chronic hepatitis B virus infection, rehospitalization, hepatocellular carcinoma, mortality, prognosis

\section{Introduction}

Chronic hepatitis B virus (HBV) infection remains a significant threat to public health, with an estimated 257 million patients and approximately 887,999 deaths worldwide. ${ }^{1}$ Chronic HBV infection may eventually lead to severe complications, such as liver cirrhosis, liver failure and hepatocellular carcinoma (HCC). ${ }^{2}$ Acute-onchronic liver failure (ACLF) is a complex syndrome characterized by rapid deterioration of liver function and underlying liver diseases, leading to multi-organ failure and high short-term mortality (50-90\%). ${ }^{3-5}$ In Asia-Pacific and Africa regions with high HBV prevalence, HBV-related ACLF (HBV-ACLF) is a critical issue that has attracted much attention from researchers. ${ }^{6}$ However, previous researches focused more on the short-term prognosis of HBV-ACLF patients, and little is known about their long-term prognosis prediction for those patients who survive over the first 6 months, such as HCC incidence and 10-year survival rate.,

Numerous risk score models have been reported to predict poor outcomes in patients with ACLF, such as albumin-bilirubin (ALBI) score, Model for End-Stage Liver Disease 
(MELD) score, and Chinese Group on the Study of Severe Hepatitis B-Acute-on-chronic Liver Failure score (COSSHACLFs), which predicted for short-term (within 6 months) mortality. ${ }^{9-14}$ However, there are few comprehensive studies on the risk model for long-term outcomes. Recently, a new risk score (aMAP score), consisting of simple and easy-to-obtain variables (age, male, albumin-bilirubin (ALBI), and platelets), was reported to predict long-term outcome (HCC occurrence) in patients with chronic hepatitis. ${ }^{15}$ Subsequently, it was also validated in chronic hepatitis $\mathrm{C}$ infected patients who achieved sustained virological response. ${ }^{16}$ Nevertheless, the predictive role of aMAP score for long-term outcomes is not well known in patients with HBV-ACLF.

Therefore, we designed this study aiming to investigate whether the aMAP score could be applicable for predicting long-term outcomes-including re-hospitalization, HCC occurrence and mortality - among patients who were diagnosed with HBV-ACLF and survived over 6 months.

\section{Methods}

\section{Patients}

It was a retrospective study approved by the Ethics Committee of Nanfang Hospital, Southern Medical University. We enrolled consecutive patients hospitalized for ACLF in our center between May 2002 and July 2012. The inclusion criteria were: patients survived over 6 months after diagnosis of ACLF. The exclusion criteria were as follows: (1) patients without chronic HBV infection; (2) patients who were younger than 12 ; (3) patients with cancer when diagnosed; (4) patients with missing data (Figure 1). Finally, a total of 82 patients meeting standards were included.

\section{Diagnostic Criteria}

According to the Asia-Pacific Association for the Study of Liver (APASL) criteria proposed in 2014, patients were reassessed and diagnosed as ACLF when meeting the following conditions: (1) pre-existing chronic liver diseases (diagnosed or undiagnosed); (2) jaundice (serum total bilirubin $\geq 5 \mathrm{mg} / \mathrm{dL}$ ); (3) coagulopathy (international normalized ratio [INR] $\geq 1.5$ ); (4) complicated with ascites or hepatic encephalopathy. ${ }^{5}$ Diagnosis of cirrhosis was based on liver biopsy and imaging findings.

\section{Data Collection and Score Calculation}

Demographic characteristics, laboratory and radiological examination results, and treatment data on the first admission were collected. As described in Fan et al's study, aMAP score was calculated at diagnosis of ACLF as follows: $(($ age $[$ year] $\times 0.06+$ gender $\times 0.89$ (male: 1 , female: 0$)+$ $0.48 \times((\log 10$ bilirubin $[\mu \mathrm{mol} / \mathrm{L}] \times 0.66)+($ albumin $[\mathrm{g} /$ L] $\times-0.085))-0.01 \times$ platelet count $\left.\left.\left[10^{3} / \mathrm{mm}^{3}\right]\right)+7.4\right) / 14.77$ $\times 100 .^{15}$

\section{Follow-Up}

All patients were followed up until November 1st, 2019 or death. The outcome events of readmission, HCC occurrence and death were collected through outpatient visits or telephone follow-up.

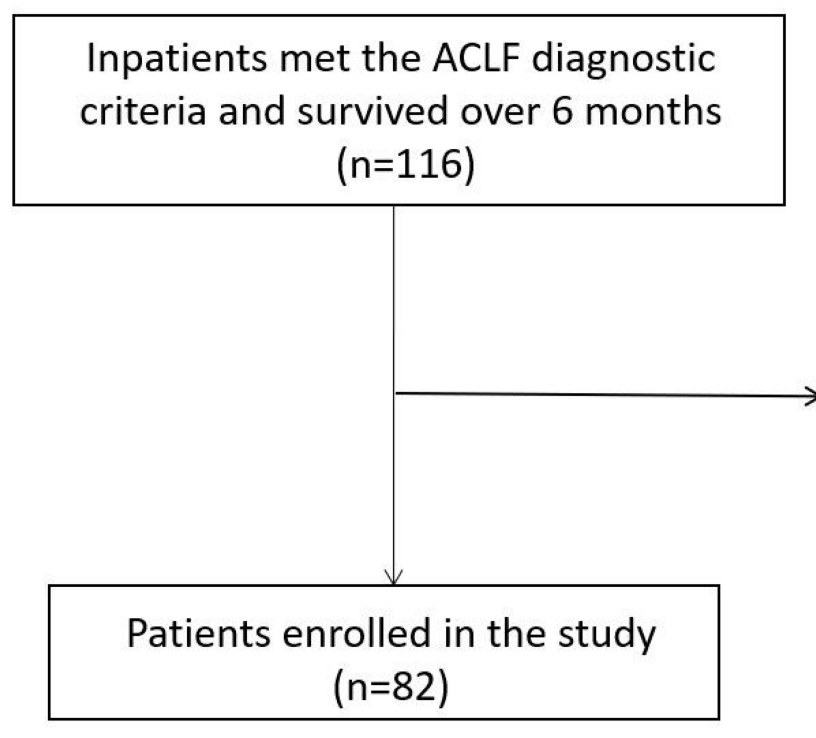

Excluded $(n=34)$ :

Age under 12 years $(n=1)$

Without chronic HBV infection ( $n=5)$

Patients with cancer $(n=2)$

Patients with missing data $(n=26)$

Figure I Flow chart of the patient enrollment.

Abbreviations: ACLF, acute on chronic liver failure; $H B V$, hepatitis $B$ virus. 
Table I. Comparison of Clinical Characteristics Between Two Groups with and without Re-Hospitalization

\begin{tabular}{|c|c|c|c|}
\hline Parameters & Without Rehospitalization ( $n=46)$ & Rehospitalization $(n=36)$ & $p$-value \\
\hline Age (years), mean (SD) & $35.7(8.2)$ & $39.4(10.5)$ & 0.087 \\
\hline Male, n (\%) & $41(89.1)$ & $32(88.9)$ & 1.000 \\
\hline Cirrhosis, n (\%) & $22(47.8)$ & $21(58.3)$ & 0.344 \\
\hline HBV DNA (log $10 \mathrm{IU} / \mathrm{mL})$, median (IQR) & $5.3(2.6)$ & $4.7(2.7)$ & 0.325 \\
\hline Creatinine $(\mu \mathrm{mol} / \mathrm{L})$, mean $(\mathrm{SD})$ & $67.4(15.6)$ & $74.5(20.2)$ & 0.076 \\
\hline INR, median (IQR) & $2.1(0.9)$ & $2.1(1.0)$ & 0.768 \\
\hline Platelet $\left(10^{9} / \mathrm{L}\right)$, mean $(\mathrm{SD})$ & I35.2 (44.9) & III.5 (59.2) & 0.042 \\
\hline aMAP score, mean (SD) & $57.1(4.9)$ & $60.5(6.6)$ & 0.011 \\
\hline Child-Pugh score, median (IQR) & $11.0(2.3)$ & $11.0(1.0)$ & 0.113 \\
\hline Child-Pugh grade & & & 0.139 \\
\hline A & $0(0)$ & $0(0)$ & \\
\hline B & II (23.9) & $4(11.1)$ & \\
\hline $\mathrm{C}$ & $35(76.1)$ & $32(88.9)$ & \\
\hline ALBI score, mean (SD) & $-1.12(0.39)$ & $-1.06(0.34)$ & 0.469 \\
\hline ALBI grade & & & 0.220 \\
\hline I & $0(0)$ & $0(0)$ & \\
\hline 2 & $13(28.3)$ & $6(16.7)$ & \\
\hline 3 & $33(7 \mid .7)$ & $30(83.3)$ & \\
\hline MELD score, median (IQR) & $22.3(5.7)$ & $22.7(8.3)$ & 0.327 \\
\hline COSSH score, mean (SD) & $6.5(0.9)$ & $6.8(1.0)$ & 0.171 \\
\hline
\end{tabular}

Abbreviation: INR, international normalized ratio.

\section{Statistical Analysis}

Continuous variables were expressed as mean \pm standard deviation or median (interquartile range, IQR) if appropriate, and were compared with $t$-test or Mann-Whitney $U$-test, respectively. Categorical variables were expressed as frequency (percentage), and compared with the chi-squared or Fisher's exact tests. Univariate and multivariate logistic regression analyses were performed to determine independent risk factors for re-hospitalization, with results presented as odds ratio (OR) and 95\% confidence interval (CI). Cox proportional hazard model analysis was performed to identify the independent risk factors of $\mathrm{HCC}$ or mortality. The incidence of $\mathrm{HCC}$ and the cumulative survival were, respectively, calculated using the Kaplan-Meier curve analysis and compared with the Log-rank test. Statistical analyses were performed using SPSS software version 26.0 (IBM Corp, Armonk, NY, USA). A two-tailed $p$-value $\leq 0.05$ was defined as statistically significant.

Table 2. Logistic Regression Analysis of Factors Associated with Re-Hospitalization

\begin{tabular}{|c|c|c|c|c|}
\hline Parameters & Univariable Analysis OR $(95 \% \mathrm{Cl})$ & $p$-value & Multivariable Analysis OR $(95 \% \mathrm{Cl})$ & $p$-value \\
\hline Age (years) & $1.044(0.995-1.097)$ & 0.081 & \multirow{12}{*}{$1.112(1.02 I-1.211)$} & \multirow{12}{*}{0.015} \\
\hline Gender (male) & $0.976(0.242-3.931)$ & 0.972 & & \\
\hline Cirrhosis & $1.527(0.634-3.68 I)$ & 0.345 & & \\
\hline HBV DNA $(\log 10 \mathrm{IU} / \mathrm{mL})$ & $0.919(0.779-1.085)$ & 0.321 & & \\
\hline Creatinine $(\mu \mathrm{mol} / \mathrm{L})$ & $1.024(0.997-\mid .05 I)$ & 0.082 & & \\
\hline INR & $0.954(0.803-1.132)$ & 0.587 & & \\
\hline Platelet $\left(10^{9} / \mathrm{L}\right)$ & $0.991(0.982-1.000)$ & 0.046 & & \\
\hline aMAP score & $1.112(1.021-1.211)$ & 0.015 & & \\
\hline Child-Pugh score & $1.324(0.954-1.837)$ & 0.093 & & \\
\hline ALBI score & $1.568(0.470-5.239)$ & 0.464 & & \\
\hline MELD score & $1.034(0.960-1.113)$ & 0.380 & & \\
\hline COSSH score & $\mathrm{I} .403(0.86 \mathrm{I}-2.286)$ & 0.175 & & \\
\hline
\end{tabular}

Abbreviation: INR, international normalized ratio. 


\section{Results}

Comparison of Clinical Characteristics
Between Two Groups with and without Re-Hospitalization

A total of 82 patients were eligible for final analysis in the study, including 46 without re-hospitalization and 36 with re-hospitalization. The median follow-up period was 105 (75.9, 134.1) months. A total of 43 patients developed cirrhosis during the follow-up. Demographic characteristics, physical examination findings and laboratory test results on the first admission were compared between the two groups and shown in Table 1. Patients with re-hospitalization had a significantly lower platelet count level than those without re-hospitalization. The aMAP score was also higher in patients with re-hospitalization.

\section{Logistic Regression Analysis to Determine Risk Factors for}

\section{Re-Hospitalization}

We conducted univariate and multivariate analyses to identify the risk factors associated with re-hospitalization. The univariate analysis revealed that lower platelet count and higher aMAP score were associated with re-hospitalization in patients with HBV-ACLF. However, on multivariate analysis, only aMAP score $(\mathrm{OR}=1.112,95 \%$ confidence interval
[CI]: 1.021-1.211, $\mathrm{p}=0.015)$ was an independent predictor of re-hospitalization (Table 2).

\section{Comparison Between HCC Occurrence and HCC Free Groups}

Among all 82 patients, 4 cases (4.9\%) of HCC occurrence were recorded. Clinical characteristics between the HCC occurrence and HCC free groups were compared and presented in Table 3. Patients in the HCC occurrence group were significantly older than HCC free groups $(p=0.022)$. The aMAP score was also significantly higher in patients with HCC occurrence $(p=0.005)$.

\section{Cox Proportional Hazard Analysis to Determine Predictors of HCC Occurrence}

The univariate analysis showed that age $(p=0.039)$ and aMAP score $(\mathrm{p}=0.046)$ were predictors of HCC occurrence in patients with HBV-ACLF (Table 4). Multivariate analysis revealed that only the aMAP was an independent risk factor of HCC occurrence (HR $=2.277,95 \%$ CI: $1.014-5.114, \mathrm{p}=$ 0.046). According to the reported definition, $45(54.9 \%)$ and $37(45.1 \%)$ patients were classified into the low $($ aMAP $<50)$ to medium (50-60) risk group and the high ( $>60)$ risk group, respectively. As shown in Figure 2, Kaplan-Meier analysis

Table 3. Comparison of Clinical Characteristics Between Two Groups with and without HCC Occurrence

\begin{tabular}{|c|c|c|c|}
\hline Parameters & Without HCC $(n=78)$ & $\operatorname{HCC}(n=4)$ & $p$-value \\
\hline Age (years), mean (SD) & $36.8(9.3)$ & $47.8(3.3)$ & 0.022 \\
\hline Male, n (\%) & $69(88.5)$ & $4(100)$ & 1.000 \\
\hline Cirrhosis, n (\%) & $40(5 \mid .3)$ & $3(75.0)$ & 0.680 \\
\hline HBV DNA $(\log 10 \mathrm{IU} / \mathrm{mL})$, median (IQR) & $5.1(2.7)$ & $4.4(3.0)$ & 0.615 \\
\hline Creatinine $(\mu \mathrm{mol} / \mathrm{L})$, mean $(\mathrm{SD})$ & $70.2(18.3)$ & $77.0(10.2)$ & 0.462 \\
\hline INR, median (IQR) & $2.1(0.9)$ & $1.6(0.2)$ & 0.055 \\
\hline Platelet (109/L), mean (SD) & $126.9(52.4)$ & $83.8(46.7)$ & 0.110 \\
\hline aMAP score, mean (SD) & $58.2(5.7)$ & $66.6(1.7)$ & 0.005 \\
\hline Child-Pugh score, median (IQR) & $11.0(2.0)$ & $11.0(2.3)$ & 0.732 \\
\hline Child-Pugh grade & & & 0.540 \\
\hline A & $0(0)$ & $0(0)$ & \\
\hline B & $15(19.2)$ & $0(0)$ & \\
\hline C & $63(80.8)$ & $4(100)$ & \\
\hline ALBI score, mean (SD) & $-1.10(0.37)$ & $-1.01(0.4 I)$ & 0.633 \\
\hline ALBI grade & & & 0.959 \\
\hline 1 & $0(0)$ & $0(0)$ & \\
\hline 2 & $18(23.1)$ & I (5.3) & \\
\hline 3 & $60(76.9)$ & $3(4.8)$ & \\
\hline MELD score, median (IQR) & $22.8(6.7)$ & $16.3(8.0)$ & 0.019 \\
\hline COSSH score, mean (SD) & $6.6(0.9)$ & $6.0(0.6)$ & 0.181 \\
\hline
\end{tabular}

Abbreviation: INR, international normalized ratio. 
Table 4. Cox Regression Analysis of Factors Associated with HCC Occurrence

\begin{tabular}{|c|c|c|c|c|}
\hline Parameters & Univariable Analysis HR $(95 \% \mathrm{Cl})$ & $p$-value & Multivariable Analysis HR ( $95 \% \mathrm{Cl})$ & p-value \\
\hline Age (years) & $1.103(1.005-1.211)$ & 0.039 & \multirow{12}{*}{$2.277(1.014-5.114)$} & \multirow{12}{*}{0.046} \\
\hline Gender (male) & $24.064(0-2|, 675| ,4|.99|)$ & 0.649 & & \\
\hline Cirrhosis & $2.363(0.244-22.898)$ & 0.458 & & \\
\hline HBV DNA ( $\log 10 \mathrm{IU} / \mathrm{mL})$ & $0.924(0.646-1.323)$ & 0.666 & & \\
\hline Creatinine $(\mu \mathrm{mol} / \mathrm{L})$ & $1.020(0.973-1.069)$ & 0.412 & & \\
\hline INR & $0.145(0.009-2.356)$ & 0.175 & & \\
\hline Platelet $\left(10^{9} / \mathrm{L}\right)$ & $0.981(0.956-1.006)$ & 0.131 & & \\
\hline aMAP score & $2.277(1.014-5.114)$ & 0.046 & & \\
\hline Child-Pugh score & $1.193(0.579-2.458)$ & 0.633 & & \\
\hline ALBI score & $2.378(0.128-44.096)$ & 0.561 & & \\
\hline MELD score & $0.793(0.621-1.013)$ & 0.064 & & \\
\hline COSSH score & $0.454(0.125-1.648)$ & 0.230 & & \\
\hline
\end{tabular}

Abbreviation: INR, international normalized ratio.

confirmed that a high-risk aMAP score was associated with HCC occurrence $(\log$-rank $p=0.027)$.

\section{Comparison of Clinical Characteristics Between Survival and Non-Survival Groups}

During the whole follow-up period, 5 deaths were recorded. Among 5 patients who died, two died of esophageal variceal rupture hemorrhage, two died of liver cancer rupture hemorrhage, and one died of end-stage liver disease. Comparisons of clinical characteristics between the survival and non-survival groups are shown in Table 5. Patients in the non-survival group had a significantly higher aMAP score than survivors $(\mathrm{p}=$ $0.013)$.

\section{Cox Proportional Hazard Analysis to Identify Predictors of Long-Term Mortality}

As depicted in Table 6, the univariate analysis showed that only aMAP score $(p=0.025)$ was a predictor of long-term mortality in patients with HBV-ACLF. Multivariate analysis confirmed that only the aMAP was an independent risk factor of 10-year mortality (HR $=1.366,95 \%$ CI: 1.040

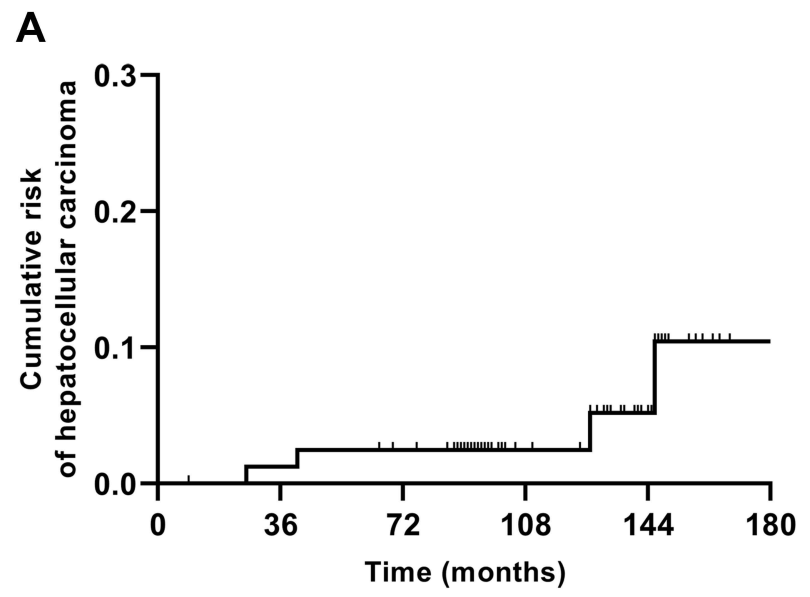

B

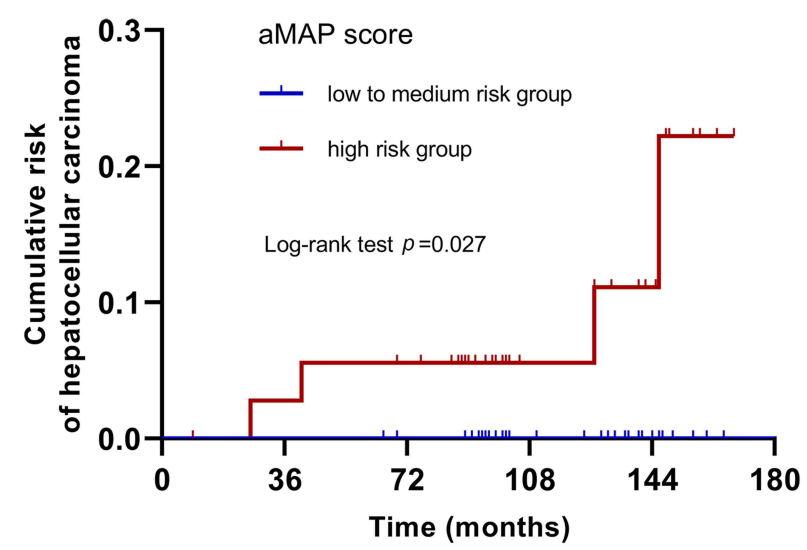

Figure 2 Cumulative risk of HCC occurrence with Kaplan-Meier analysis. (A) Cumulative risk of HCC occurrence in all patients with HBV-ACLF; (B) cumulative risk of $\mathrm{HCC}$ occurrence according to the aMAP scores stratification. Kaplan-Meier analysis confirmed that a high-risk aMAP score was associated with HCC occurrence (log-rank $p=0.027)$.

Abbreviation: HCC, hepatocellular carcinoma. 
Table 5. Comparison of Clinical Characteristics Between Survival Group and Non-Survival Group

\begin{tabular}{|c|c|c|c|}
\hline Parameters & Survival $(n=77)$ & Non-Survival $(n=5)$ & $p$-value \\
\hline Age(years), mean (SD) & $36.8(9.4)$ & $45.0(5.9)$ & 0.060 \\
\hline Male, n (\%) & $69(89.6)$ & $4(80.0)$ & 0.450 \\
\hline Cirrhosis, n (\%) & $38(49.4)$ & $5(100)$ & 0.083 \\
\hline HBV DNA (log $10 \mathrm{IU} / \mathrm{mL})$, median (IQR) & $5.1(2.7)$ & $4.4(2.7)$ & 0.580 \\
\hline Creatinine $(\mu \mathrm{mol} / \mathrm{L})$, mean $(\mathrm{SD})$ & $70.5(18.5)$ & $70.8(5.0)$ & 0.969 \\
\hline INR, median (IQR) & $2.1(1.0)$ & $2.1(0.7)$ & 0.970 \\
\hline Platelet (I09/L), mean (SD) & $127.5(52.8)$ & $82.7(30.8)$ & 0.065 \\
\hline aMAP score, mean (SD) & $58.2(5.8)$ & $64.9(3.0)$ & 0.013 \\
\hline Child-Pugh score, median (IQR) & $1 \mathrm{I} .0(2.0)$ & $12.0(2.0)$ & 0.686 \\
\hline Child-Pugh grade & & & 0.485 \\
\hline$A$ & $0(0)$ & $0(0)$ & \\
\hline B & $15(19.5)$ & $0(0)$ & \\
\hline C & $62(80.5)$ & $5(100)$ & \\
\hline ALBI score, mean (SD) & $-1.11(0.36)$ & $-0.83(0.36)$ & 0.095 \\
\hline ALBI grade & & & 0.373 \\
\hline 1 & $0(0)$ & $0(0)$ & \\
\hline 2 & $19(24.7)$ & $0(0)$ & \\
\hline 3 & $58(75.3)$ & $5(100)$ & \\
\hline MELD score, median (IQR) & $22.5(6.1)$ & I8.8 (II.I) & 0.509 \\
\hline COSSH score, mean (SD) & $6.6(1.0)$ & $6.5(0.9)$ & 0.711 \\
\hline
\end{tabular}

Abbreviation: INR, international normalized ratio.

1.794, $\mathrm{p}=0.025)$. As is shown in Figure 3, Kaplan-Meier analysis showed that a high-risk aMAP score was associated with worse survival (log-rank $\mathrm{p}=0.012$ ).

\section{Discussion}

Limited previous researches focused on risk score models for predicting long-term outcomes of HBV-ACLF patients. In this study, aMAP score was identified as an independent risk factor of long-term re-hospitalization, HCC occurrence and long-term mortality in patients who survived HBV-ACLF over the first 6 months. The aMAP score with high risk $(>60)$ was significantly associated with higher risk of HCC occurrence and long-term mortality.

It was reported by Chen et al that cumulative incidence rates of hepatocellular carcinoma were up to $14.9 \%{ }^{17}$ Compared with previous studies, the HCC occurrence and mortality rate were lower in this study, which could be attributed to the following reasons. On the one hand, most previous studies focused on short-term prognosis, while ACLF patients have a high risk of mortality at the early period (with 6 months after diagnosis). However, this study aimed to investigate the long-term prognosis, so only patients who can survive over the first 6 months were included, which led to a lower mortality. On the other hand, patients in this study started antiviral therapy before or at the time of diagnosis of HBV-ACLF. Several studies reported that nucleoside analogs improved the long-term outcomes of HBV-ACLF patients. ${ }^{18,19}$

Due to the dangerous condition and high short-term mortality of ACLF, many researchers were committed to improving its short-term prognosis. Currently, there are few studies on the long-term prognosis of HBV-ACLF. Baseline data in this study showed that patients who survived over the first 6 months had a good long-term prognosis. However, because of the poor prognosis and high treatment cost of liver complications, it is still necessary to explore simple indicators for risk stratification to adjust the treatment and improve the prognosis for high-risk patients. Previous studies have reported several well-established prognostic models of liver diseases, such as Child-Pugh score and MELD score, which were mainly applicable for short-term prognosis evaluation. ${ }^{20}$ However, this study focused on the long-term prognosis of ACLF patients and the prognostic scores above were also included in the analysis.

The recently reported aMAP score was a long-term prognostic model aiming to predict the HCC occurrence in patients with chronic hepatitis. The aMAP score consisted of 4 simple and easy-to-obtain variables: age, male, ALBI, and platelet. ALBI consisted of albumin and 
Table 6. Cox Regression Analysis of Factors Associated with Mortality

\begin{tabular}{|c|c|c|c|c|}
\hline Parameters & Univariable Analysis HR $(95 \% \mathrm{Cl})$ & p-value & Multivariable Analysis HR ( $95 \% \mathrm{Cl})$ & p-value \\
\hline Age (years) & I.082 (0.995-I.I77) & 0.064 & \multirow{12}{*}{$1.366(1.040-1.794)$} & \multirow{12}{*}{0.025} \\
\hline Gender (male) & $0.475(0.053-4.25 \mathrm{I})$ & 0.505 & & \\
\hline Cirrhosis & $57.729(0.042-80,017.229)$ & 0.272 & & \\
\hline HBV DNA (log $10 \mathrm{IU} / \mathrm{mL})$ & $0.916(0.666-1.258)$ & 0.586 & & \\
\hline Creatinine $(\mu \mathrm{mol} / \mathrm{L})$ & $1.001(0.955-1.049)$ & 0.962 & & \\
\hline INR & $0.846(0.27 I-2.646)$ & 0.774 & & \\
\hline Platelet $\left(10^{9} / \mathrm{L}\right)$ & $0.979(0.956-1.002)$ & 0.074 & & \\
\hline aMAP score & $1.366(1.040-1.794)$ & 0.025 & & \\
\hline Child-Pugh score & $1.558(0.756-3.208)$ & 0.229 & & \\
\hline ALBI score & II.304 (0.664-192.336) & 0.094 & & \\
\hline MELD score & $0.952(0.806-1.124)$ & 0.562 & & \\
\hline COSSH score & $0.865(0.322-2.324)$ & 0.774 & & \\
\hline
\end{tabular}

Abbreviation: INR, international normalized ratio.

bilirubin and was initially developed to assess liver function and predict prognosis in HCC patients. ${ }^{11,21}$ Platelets also play an essential role in chronic liver diseases. For example, the platelet count decreased with the progression of liver fibrosis. ${ }^{22,23}$ Therefore, aMAP score can reflect the liver function objectively and be associated with the outcomes of liver diseases. However, the predictive value of the score is unknown for patients with HBV-ACLF. Therefore, we validated whether aMAP score was applicable for predicting readmission, HCC occurrence and long-term mortality in HBV-ACLF patients who survived over the first 6 months. The current study revealed that aMAP score was an independent predictor of all outcome events above. Furthermore, aMAP stratification can further identify patients with high-risk scores $(>60)$ associated with a high HCC occurrence and long-term mortality rate. All non-survivors in the study were confirmed to have high-risk scores. Therefore, high-risk HBV-ACLF patients could be identified early through aMAP score and intervened timely and appropriately.

This was a study with follow-up over 10 years and confirmed that aMAP score was a risk indicator of longterm outcomes in patients with HBV-ACLF. Nevertheless, the study still has limitations because of its retrospective and single-center design. Moreover, this study was limited by the small number of HCC cases and deaths. Therefore, a prospective and multi-center study with larger sample size was warranted to validate the predictive role of aMAP score further.
A

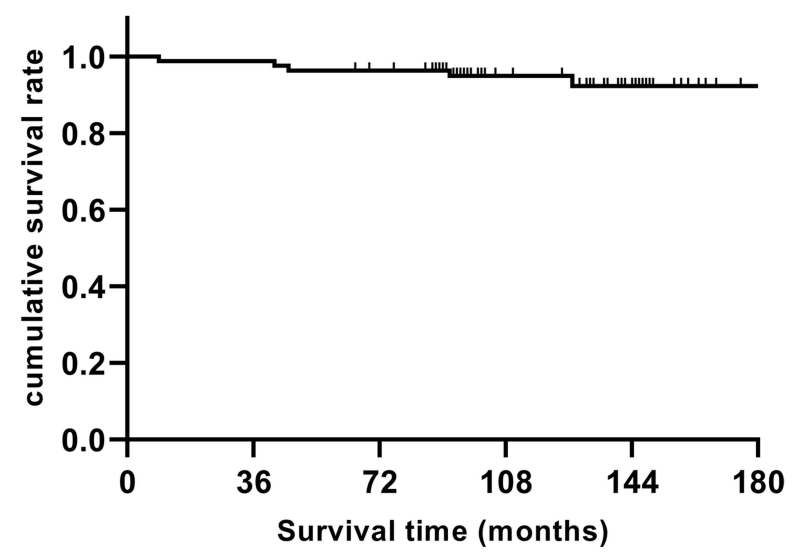

B

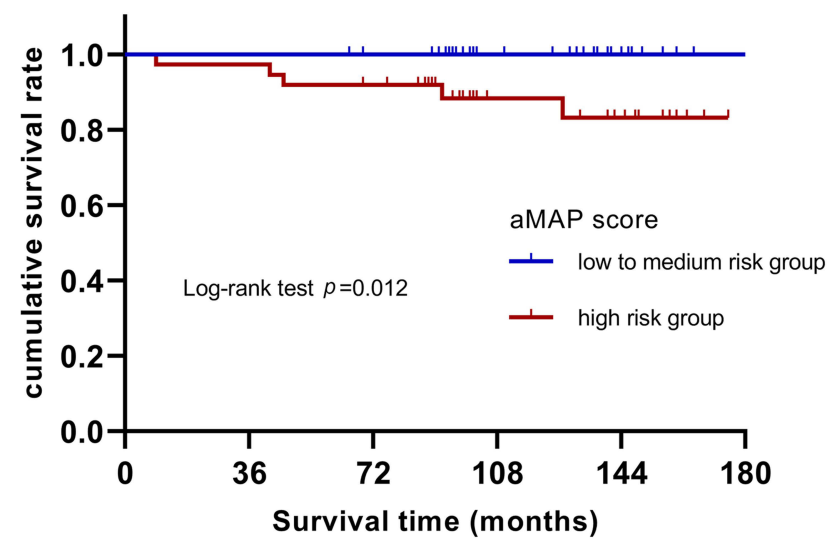

Figure 3 Cumulative survival rate with Kaplan-Meier analysis. (A) Cumulative survival rate analysis in all patients with HBV-ACLF; (B) cumulative survival rate analysis according to the aMAP scores stratification. Kaplan-Meier analysis showed that a high-risk aMAP score was associated with worse survival $($ log-rank $\mathrm{P}=0.0 \mathrm{I} 2$ ). 


\section{Conclusions}

In summary, for patients with HBV-ACLF, aMAP score was an independent risk indicator of re-hospitalization, HCC occurrence and long-term mortality. High-risk score patients were associated with a high rate of HCC incidence and mortality. Clinicians should pay more attention to patients with high-risk scores and adjust treatments strategies appropriately.

\section{Data Sharing Statement}

The data used in the current study are available from the corresponding authors upon reasonable request.

\section{Ethical Approval and Consent to Participate}

This study was approved by the Ethics Committee of Nanfang Hospital, Southern Medical University. It was a retrospective study and the data were anonymous, so the requirement for written informed consent was waived. Patient data confidentiality was guaranteed, and all procedures were conducted following the Declaration of Helsinki.

\section{Funding}

This work was supported by the National Natural Science Foundation of China (grant no. 81971949).

\section{Disclosure}

Yunqing Sun and Zhuohong Li are co-first authors for this study. The authors declare no conflicts of interest.

\section{References}

1. World Health Organization. Global Hepatitis Report 2017. World Health Organization; 2017.

2. Collaborators GBDCoD. Global, regional, and national age-sexspecific mortality for 282 causes of death in 195 countries and territories, 1980-2017: a systematic analysis for the Global Burden of Disease Study 2017. Lancet. 2018;392(10159):1736-1788. doi:10.1016/S0140-6736(18)32203-7

3. Moreau R, Jalan R, Gines P, et al. Acute-on-chronic liver failure is a distinct syndrome that develops in patients with acute decompensation of cirrhosis. Gastroenterology. 2013;144(7):1426-U1189. doi:10.1053/j.gastro.2013.02.042

4. Polson J, Lee WM; American Association for the Study of Liver D. AASLD position paper: the management of acute liver failure. Hepatology. 2005;41(5):1179-1197. doi:10.1002/hep.20703

5. Sarin SK, Kedarisetty CK, Abbas Z, et al. Acute-on-chronic liver failure: consensus recommendations of the Asian Pacific Association for the Study of the Liver (APASL) 2014. Hepatol Int. 2014;8 (4):453-471. doi:10.1007/s12072-014-9580-2

6. Zhao RH, Shi Y, Zhao H, Wu W, Sheng JF. Acute-on-chronic liver failure in chronic hepatitis B: an update. Expert Rev Gastroenterol Hepatol. 2018;12(4):341-350. doi:10.1080/17474124.2018.1426459
7. Wu DX, Zhang SN, Xie ZY, et al. Plasminogen as a prognostic biomarker for HBV-related acute-on-chronic liver failure. $J$ Clin Invest. 2020;130(4):2069-2080. doi:10.1172/JCI130197

8. Yan $\mathrm{H}, \mathrm{Wu} \mathrm{W}$, Yang Y, Wu Y, Yang Q, Shi Y. A novel integrated Model for End-Stage Liver Disease model predicts short-term prognosis of hepatitis B virus-related acute-on-chronic liver failure patients. Hepatol Res. 2015;45(4):405-414. doi:10.1111/hepr.12362

9. Kamath PS, Wiesner RH, Malinchoc M, et al. A model to predict survival in patients with end-stage liver disease. Hepatology. 2001;33 (2):464-470. doi:10.1053/jhep.2001.22172

10. Wu T, Li J, Shao L, et al. Development of diagnostic criteria and a prognostic score for hepatitis B virus-related acute-on-chronic liver failure. Gut. 2018;67(12):2181-2191. doi:10.1136/gutjnl-2017314641

11. Feng $\mathrm{D}$, Wang $\mathrm{M}$, $\mathrm{Hu} \mathrm{J}$, et al. Prognostic value of the albumin-bilirubin grade in patients with hepatocellular carcinoma and other liver diseases. Ann Transl Med. 2020;8(8):553. doi:10.21037/atm.2020.02.116

12. Mohammed MAA, Khalaf MH, Liang T, et al. Albumin-bilirubin score: an accurate predictor of hepatic decompensation in high-risk patients undergoing transarterial chemoembolization for hepatocellular carcinoma. J Vasc Interv Radiol. 2018;29(11):1527-1534 e1521. doi:10.1016/j.jvir.2018.06.016

13. Wu DX, Sun ZY, Liu XL, et al. HINT: a novel prognostic model for patients with hepatitis $\mathrm{B}$ virus-related acute-on-chronic liver failure. Aliment Pharm Ther. 2018;48(7):750-760. doi:10.1111/apt.14927

14. Zheng YX, Zhong X, Li YJ, Fan XG. Performance of scoring systems to predict mortality of patients with acute-on-chronic liver failure: a systematic review and meta-analysis. $J$ Gastroen Hepatol. 2017;32(10):1668-1678. doi:10.1111/jgh.13786

15. Fan R, Papatheodoridis G, Sun J, et al. aMAP risk score predicts hepatocellular carcinoma development in patients with chronic hepatitis. $J$ Hepatol. 2020;73(6):1368-1378. doi:10.1016/j. jhep.2020.07.025

16. Yamashita Y, Joshita S, Sugiura A, et al. aMAP score prediction of hepatocellular carcinoma occurrence and incidence-free rate after a sustained virologic response in chronic hepatitis C. Hepatol Res. 2021;51(9):933-942. doi:10.1111/hepr.13689

17. Chen CJ, Yang HI, Su J, et al. Risk of hepatocellular carcinoma across a biological gradient of serum hepatitis B virus DNA level. $J$ Am Med Assoc. 2006;295(1):65-73. doi:10.1001/jama.295.1.65

18. Cui YL, Yan F, Wang YB, et al. Nucleoside analogue can improve the long-term prognosis of patients with Hepatitis B virus infection-associated acute on chronic liver failure. Digest Dis Sci. 2010;55(8):2373-2380. doi:10.1007/s10620-010-1257-7

19. Philips CA, Sarin SK. Potent antiviral therapy improves survival in acute on chronic liver failure due to hepatitis B virus reactivation. World J Gastroenterol. 2014;20(43):16037-16052. doi:10.3748/wjg. v20.i43.16037

20. D'Amico G, Garcia-Tsao G, Pagliaro L. Natural history and prognostic indicators of survival in cirrhosis: a systematic review of 118 studies. $J$ Hepatol. 2006;44(1):217-231. doi:10.1016/j. jhep.2005.10.013

21. Johnson PJ, Berhane S, Kagebayashi C, et al. Assessment of liver function in patients with hepatocellular carcinoma: a new evidence-based approach-the ALBI grade. J Clin Oncol. 2015;33 (6):550-558. doi:10.1200/JCO.2014.57.9151

22. Kurokawa T, Ohkohchi N. Platelets in liver disease, cancer and regeneration. World J Gastroenterol. 2017;23(18):3228-3239. doi:10.3748/wjg.v23.i18.3228

23. Lemoine M, Shimakawa Y, Nayagam S, et al. The gamma-glutamyl transpeptidase to platelet ratio (GPR) predicts significant liver fibrosis and cirrhosis in patients with chronic HBV infection in West Africa. Gut. 2016;65(8):1369-1376. doi:10.1136/gutjnl-2015-309260 


\section{Publish your work in this journal}

The International Journal of General Medicine is an international, peer-reviewed open-access journal that focuses on general and internal medicine, pathogenesis, epidemiology, diagnosis, monitoring and treatment protocols. The journal is characterized by the rapid reporting of reviews, original research and clinical studies

across all disease areas. The manuscript management system is completely online and includes a very quick and fair peer-review system, which is all easy to use. Visit http://www.dovepress.com/ testimonials.php to read real quotes from published authors. 